\title{
KESTING Marietta. - Affective Images, Post-apartheid Documentary Perspectives
}

New York, SUNY Press, 2017, 278 p., bibl.

\section{Katja Gentric}

\section{(2) OpenEdition}

\section{Journals}

Édition électronique

URL : http://journals.openedition.org/etudesafricaines/22197

DOI : 10.4000/etudesafricaines.22197

ISSN : 1777-5353

Éditeur

Éditions de l'EHESS

Édition imprimée

Date de publication : 1 juin 2018

Pagination : 552-557

ISBN : 978-2-7132-2742-4

ISSN : 0008-0055

Référence électronique

Katja Gentric, « Kesting Marietta. - Affective Images, Post-apartheid Documentary Perspectives », Cahiers d'études africaines [En ligne], 230 | 2018, mis en ligne le 01 juin 2018, consulté le 06 janvier 2021. URL http://journals.openedition.org/etudesafricaines/22197 ; DOI : https://doi.org/10.4000/ etudesafricaines. 22197

Ce document a été généré automatiquement le 6 janvier 2021.

(c) Cahiers d'Études africaines 


\title{
KESTING Marietta. - Affective Images, Post-apartheid Documentary Perspectives
}

New York, SUNY Press, 2017, 278 p., bibl.

\author{
Katja Gentric
}

\section{RÉFÉRENCE}

KESTING Marietta. - Affective Images, Post-apartheid Documentary Perspectives. New York, SUNY Press, 2017, 278 p., bibl.

1 Une recherche sur les médias documentaires issus du contexte sud-africain, interrogés dans une perspective historique et politique, était très attendue, pour ne pas dire qu'il était urgent que ce livre soit écrit. En l'accolant à la question des "affective images", Marietta Kesting saisit par ce texte deux préoccupations phares actuelles et les interroge l'une au prisme de l'autre.

2 Les contributions analysant le retour de l'affect parmi les théories des médias s'accumulent depuis le début des années 1990. Nombreux sont les auteurs, comme Marietta Kesting, qui reconnaissent l'utilisation initiale du concept par Gilles Deleuze et Felix Guattari ${ }^{6}$. Kesting reconnait également la contribution de Brian Massumi et son travail de théorisation de l'affect dans le contexte des médias virtuels ${ }^{7}$. Elle ajoute à ce corpus théorique la remarque que les critiques féministes pressentaient une forme de recherche de l'affect par l'image avant qu'elle soit devenue un sujet en vogue (p. 11). En se montrant sensibles aux relations qu'entretient le spectateur avec les médias, les analyses féministes complexifiaient des catégorisations dualistes entre corps et esprit et interrogeaient la part affective de cette rencontre ${ }^{8}$. Kesting revendique à son tour la possibilité d'un sens de la vision qui aurait pris corps (" embodied seeing») qui va de pair avec, mais ne sera jamais remplacé par un regard analytique (" analytical gaze»). Quoi qu'il en soit, quant à l'origine de la notion, la question des affects et des percepts 
mobilisés dans la production et la réception des médias est pressante : tous observent le flux des images rendu possible par l'omniprésence des nouveaux médias s'étant accélérés à une vitesse vertigineuse. Il appartient aux « professionnels de l'image et des médias audio-visuels » (artistes, cinéastes, concepteurs de sites web, historiens d'art, activistes, spécialistes des visual studies, ou des media studies, etc.) de mettre des mots sur la fascination que les médias exercent sur leurs utilisateurs. Il leur faut travailler pour créer les conditions d'un devenir lucide quant au fonctionnement et également aux effets des médias sur les habitudes du quotidien, sur les avis politiques, sur les décisions commerciales. Ils doivent également savoir analyser en quoi ces médias reflètent, soutiennent et perpétuent des stéréotypes et des discriminations sociales. Ces influences (intuitivement perçues et souvent non admises) passent par la charge affective que les médias exercent sur le regardeur et/ou l'auditeur.

Deuxième question pressante posée par l'étude de l'effet de l'affectif sur l'utilisateur des médias est : « qu'est-ce qui nous pousse à l'action?». En parlant d'images qui ont vu le jour dans des situations humaines plus que difficiles («crises» de réfugiés, de migrants, de xénophobie, de brutalité policière, de guerre), la préoccupation n'est plus seulement celle du voyeurisme et de la curiosité quasi obscène que le public porte à ces images sensationnelles. Le professionnel des médias doit également être à même d'estimer l'impact que ces médias auront sur l'imagination sociale. Cette question est un sujet brûlant dans nos façons d'appréhender l'écosystème socio-politique mondial ou local. Il peut s'agir d'un impact positif : l'utilisateur peut se sentir touché au point de passer à une forme d'action constructive, ou il peut s'agir d'une réaction affective négative. L'affect exercé par les médias est une thématique beaucoup débattue et les nouvelles contributions à ce débat doivent s'attendre à être scrutées par un lectorat très exigeant.

4 Affective images de Marietta Kesting se décline dans ses rapports à l'histoire récente de l'Afrique du Sud. Même s'il s'agit là du premier ouvrage qui combine ces deux thématiques précises, l'auteure a pu prendre appui sur des précurseurs tels que Patricia Hayes 9 qui a écrit une histoire sociale à partir d'analyses d'images documentaires et de Kylie Thomas qui a signé un article au titre parlant "Wounding Apertures $»^{10}$. Kesting construit son texte habilement dans un dialogue entre ses deux sources.

5 L'introduction expose ses partis pris dans une description détaillée de la rencontre entre l'auteure et une photographie des manifestations contre les laissez-passer ${ }^{11} \mathrm{du}$ début des années 1960, prise par Ian Berry, un photographe de Magnum Photos. Par la description minutieuse de l'image et de toutes les conditions de sa migration jusque sa nouvelle publication dans ce livre sur « cette page ", vue « ici ", « aujourd'hui » par le lecteur. Kesting introduit une par une les questions que l'ouvrage cherchera à élucider. Il y est question de la signification de la photographie d'identité, de la résistance contre la façon dont une autorité peut vouloir imposer une certaine forme de représentation et donc une identité, de l'historique dans lequel s'inscrit le moment et le lieu précis de la prise de vue, ainsi que des questions de genre dans les pratiques de documentation. En brûlant leurs laissez-passer, les hommes sur la photographie s'engagent à détruire une forme d'(auto)portrait imposée par un pouvoir politique despotique, une action que Kesting interprète comme une forme de performance affective. Kesting mettra ensuite cette image en lien avec d'autres images célèbres de personnalités brûlant leurs laissez-passer et avec la thématique du feu dans d'autres situations violentes. 
6 Lors de son enquête, M. Kesting cherchera à faire face à deux photographies des plus difficiles à regarder. Ainsi, l'auteure obligera ses lecteurs à faire de même. Au-delà de l'image de Sam Nzima prise à Soweto en 1976, maintes fois discutée et analysée, Kesting montre, chacune dans un contexte différent, deux images d'un homme en agonie alors brûlé vif par les membres d'une foule. La première date de 1990 lorsque des supporters de l'ANC exécutaient en public dans les townships des individus qu'ils soupçonnaient de collaborer avec le régime et donc d'être des traîtres à la lutte contre l'apartheid. La deuxième image date de 2008 et montre la victime d'une attaque xénophobe fortement médiatisée. Kesting se réfère à ces photographies comme des viols par l'image («violating images», p. 46) puisqu'elles ne montrent que la surface de l'événement, créant ainsi un sentiment de choc. De cette mise en parallèle entre 1990 et 2008 découle un double argument. Premièrement, Kesting soutient qu'un fait contemporain ne peut pas être compris sans sa perspective historique. Deuxièmement, la chercheuse suit la trace du premier incident dans d'autres exemples glanés d'un ensemble de pratiques artistiques, de documentaires, de fictions, de publicités, qui toutes opèrent à leur façon un travail de mémoire suite à ces moments de terreur. Elle montre à travers cet enchaînement les répercussions du premier incident et comment les images produites ensuite cherchent à mieux cerner l'affect immédiat d'un moment traumatique. Ainsi, ces constructions médiatiques opèrent la migration de la charge affective dégagée par l'événement initial. Par la mise en parallèle de ces deux images et par leurs reprises cherchant à les dépasser, la question de l'identité opprimée des années 1960 est devenue pertinente pour la situation des migrants de l'Afrique du Sud postapartheid. Dans l'imagination populaire, les attaques xénophobes sont mises en perspective avec le système de «migrant labour » (représenté par les laissez-passer) sous la législation de l'apartheid.

7 Toutefois, notre critique positive quant au parti pris de privilégier l'inscription du phénomène social dans sa dimension historique ne serait pas complète si elle ne mentionnait pas une inattention gênante. Quelques imprécisions se glissent dans le texte de Kesting, et quelques approximations sur la chronologie dans laquelle les lois de l'apartheid ont pris effet (pp. 20-21) rendent difficile pour le lecteur la différenciation des lois coloniales de celles de l'État de l'apartheid sous le gouvernement nationaliste. Quelques confusions sur les dates des manifestations de Sharpeville et de Soweto ${ }^{12}$ sont également à souligner. Insister sur cet aspect peut paraître tatillon, mais ceci avertit le lecteur quant à d'autres imprécisions. La légende de la première image de l'ouvrage et les informations que Kesting reprend dans son texte indiquent que la photographie fut prise à Soweto en 1961. Or, la dénomination de Soweto n'est pas utilisée avant 1963, même si les townships qui seront regroupés sous ce nom en 1963 existaient bien déjà en 1961. À quel moment cette imprécision s'est-elle glissée dans la chaîne d'informations ? $\mathrm{Au}$ moment où Ian Berry transmet l'image à l'agence Magnum? Au moment où Magnum réorganise ses prises de vue selon des événements phares de la lutte contre l'apartheid? S'agirait-il du détournement d'une image en tant qu' "arme " qui se justifierait par l'urgence de sa lutte? (Kesting parle de l'image documentaire en tant que "weapon in the struggle», pp. 190-192). Ce détail d'une éventuelle confusion négligente sur les dates des massacres de Soweto et de Sharpeville aurait-il une quelconque importance pour son argument en général ? En effet, ces approximations pourraient avoir peu d'importance dans le cadre d'une thèse de doctorat portant sur l'image médiatique dans son contexte contemporain. Pourtant, ces imprécisions risquent de poser des problèmes aux lecteurs de l'ouvrage, car ce livre s'imposera sans 
doute comme référence dans les années à venir. Dans une autre perspective, il est aussi concevable que cette imprécision s'avère être une lacune féconde puisqu'elle montre à quel point les personnes à qui Kesting a parlé, et avec qui elle a établi une relation de confiance, n'ont pas pu faire le travail de mémoire nécessaire afin de pouvoir distinguer clairement les deux massacres, commis pourtant à seize ans d'intervalle et dans deux localités géographiquement distinctes. S'agirait-il ici d'un symptôme de l'efficacité de la propagande de l'État de l'apartheid que d'avoir réussi à effacer des mémoires ces seize ans de black-out total entre les deux dates? C'est une raison de plus de l'urgence de ce livre. Il s'avère que le flou des circonstances précises des années d'apartheid qui se trahit dans les incertitudes sur les dates devient l'illustration même de la remarque de Santu Mofokeng relayé par Patricia Hayes « There is no vocabulary for the non-photographed of apartheid $»^{13}$.

La sensibilité au détail est d'ailleurs un constituant fondamental du texte de Kesting, presque comme si elle voulait secourir des détails rescapés. Dès la description de la première image des hommes brûlant leur laissez-passer, Marietta Kesting fait le parallèle entre un détail technique $d u$ vocabulaire photographique avec tout le processus de migration de signification par l'image et par l'imaginaire qu'elle opérera par la suite. Elle fait remarquer que l'objectif utilisé par Ian Berry serait un téléobjectif de type "burning lense». Kesting (entre autres p.194) utilise cette métaphore de manière constante dans son ouvrage: "Understanding the violence of the present through the (lens of the) violence of the past ».

9 Le feu et sa très haute charge affective ("affective index») créent une relation diachronique entre des moments historiques. Kesting parvient à montrer le mutisme des victimes dans ce type de représentation et l'urgence de redevenir acteur. Pour cela, elle théorise une catégorie médiatique qu'elle qualifie de "photographic speech acts». Il s'agit de projets menés par des artistes photographes permettant aux migrants d'avoir un contrôle sur l'image qui les représentera dans l'imaginaire collectif. Certains de ces projets sont de véritables lieux de photographie participative alors que d'autres réitèrent les stéréotypes et enferment les sujets dans des clichés. Un deuxième cas d'étude porte sur les pratiques de reenactment. Des artistes contemporains tels que Thenjiwe Niki Nkosi et le collectif du Center for Historical Reenactments ont expérimenté cette pratique en tant que méthode afin de se réapproprier l'histoire là où les documents font défaut. Or, la technique de faire rejouer une scène importante afin de produire une image est une pratique commune des régisseurs de documentaires. Kesting soumet ces différentes formes à une fine analyse des glissements qui s'installent dans cette suite de pratiques de médiatisation. Le troisième vecteur pertinent de l'argument est le droit de rester in/visible et les formes sous lesquelles cette in/visibilité peut devenir parlante.

10 À travers cette suite d'analyses, Kesting met en place ce qu'elle nomme une « responsible spectatorship » (p. 89), une éthique que l'auteure cherche à respecter à tout prix. Le spectateur conscient saura faire la différence entre le voyeurisme et le degré d'immersion imaginaire nécessaire afin de se laisser toucher, de se laisser impressionner par ce qu'une image rend visible ou une production sonore rend audible. Autant dans la production que dans la réception, les réflexions aboutissent au droit de l'individu de se faire auteur de l'image qui représentera son «moi» et la lucidité nécessaire quant à la relation inévitable entre affect et percept, entre affect et politique. Les sons ou images (mouvantes ou fixes) par lesquels les médias s'adressent 
au public se déclinent en différents modes, différentes intensités d'affect et de percept. Dans le même mouvement se crée une oscillation entre passé et présent (p.14). Le parallélisme entre migration des individus et migration d'une charge qui part d'un événement et l'accompagne à travers plusieurs médias ou phases historiques est considéré comme un processus complexe d'influences mutuelles. Les images sont perçues comme faisant partie d'un réseau d'acteurs vivants ou inanimés, d'affects, de modes de transmission et de visionnage partiellement déterminés par des contraintes et des possibilités techniques (p. 7).

11 Pourquoi ce livre était si attendu? Marietta Kesting en a identifié la raison : dans la situation sud-africaine contemporaine, il existe, en termes de production d'images, une coïncidence unique entre plusieurs facteurs. Tout d'abord, une conscience aiguë de l'histoire récente (p. 191), avec toutes ses contradictions et tous ses rebondissements. Ensuite, des professionnels de l'image (p.183) dotés d'une volonté de développer le vocabulaire esthétique et éthique nécessaire afin de pouvoir aborder ces questions difficiles et répondre à l'urgence avec laquelle on cherche un imaginaire qui sera capable de véhiculer des situations complexes et inexpliquées.

\section{NOTES}

6. G. DELeUze \& F. GUATTARI, chapitre «Percept, affect et concept ", in Qu'est-ce que la philosophie?, Paris, Éditions de Minuit, 1991.

7. B. Massumi, Parables for the Virtual: Movement, Affect, Sensation, Durham, Duke University Press, 2002.

8. A. KOIVUNEN, «An Affective Turn? Reimagining the Subject of Feminist Theory ", in M. LILJESTRÖM \& S. PAASONEN (eds.), Working with Affect in Feminist Readings: Disturbing Differences, London-New York, Routledge, 2010, pp. 8-28.

9. P. HAYES, «Power, Secrecy, Proximity: A Short History of South African Photography », Kronos, 33, November 2007, pp. 139-162.

10. K. THомAS, «Wounding Apertures: Violence, Affect and Photography during and after Apartheid », Kronos, 38 (1), 2012, pp. 204-218.

11. Un pass «muet » renforçant le système de ségrégation sur le territoire de la République sud-africaine.

12. Dans le texte p. 26 et dans la note 47 de la conclusion (p.242). Ailleurs, les massacres de Sharpeville et de Soweto sont respectivement liés à la bonne date (pp. 36, $39,125,171$; note 26, p. 214), mais il n'est pas dit clairement qu'il s'agit de deux incidents distincts.

13. P. HAYES, "Santu Mofokeng, Photographs: "The Violence is in the Knowing" ", History and Theory, 48, 2009, p. 43 (cité par Kesting, p. 8). 